\title{
Lagrangian Mechanics Application for Determination of Reentry Rate of Low Earth Orbiting Satellite
}

\author{
Pawan Prakash Dabeer ${ }^{+*}$ and Sagar B ManeDeshmukh ${ }^{\dagger}$ \\ †Department of Mechanical Engineering, Savitribai Phule Pune University, Pune, India
}

Accepted 01 Sept 2016, Available online 02 Sept 2016, Vol.6, No.5 (Oct 2016)

\begin{abstract}
This paper addresses the application of Lagrangian Mechanics for the determination of time required for reentry of the low orbiting satellites into the earth's atmosphere. The Lagrangian approach takes gravitational force, drag force and kinetic energy into account for reentry of low orbiting satellite. Lagrangian mechanics investigates the effect of atmospheric drag on the motion of satellites in low Earth orbits. Investigation of reentry enables to determine the intervals when the satellite is to be fired back to its original orbit. The Cartesian coordinate system and polar coordinate system is used as reference frame for writing equations of motion then it is transformed to general coordinate system for application of Lagrangian equation of motion. The similar principle can be applied for the determination of reentry of space junk and low orbiting satellites and the prior risk on earth because of reentry of the same can be examined and controlled. It is important to determine the reentry rate of satellite so that it functions its desired application efficiently when it is orbiting at the predefined orbit at the time of its launch.
\end{abstract}

\section{Keywords: Lagrangian Equation, Reentry Rate, Low Earth Orbiting Satellite.}

\section{Introduction}

\subsection{Reentry of Satellite}

Many satellites do not remain in their orbits life long, but gradually come closer to Earth. This is because Earth's atmosphere does not end eventually, but becomes progressively thinner at higher altitudes. In fact, there is still some atmosphere several hundred kilometers from Earth's surface, where some satellites orbit Earth. Because the atmosphere is very thin at those high altitudes, satellites can take a long time to come down.

Satellites orbiting around the Earth orbit at hundreds of kilometers in altitude; it may take years or tens of years to return to Earth. Satellites at higher altitude are of less concern with respect to reentry hazards because they can stay in orbit for hundreds to even thousands of years.

Satellite loses its altitude when it enters denser regions of the atmosphere. Friction and compression generates a great amount of heat. This is because of the high velocity of orbiting satellites. The tremendous amount of heat generated is sufficient to melt or vaporize the entire satellite or portions of the satellite. The similar phenomenon is noticed during a meteor shower, where streaks of light (meteors or shooting

*Corresponding author: Pawan Prakash Dabeer stars) are generated by meteoroids they burn up in the atmosphere.

Although most of satellites burn up completely during atmospheric reentry, some satellite components can and do survive the reentry heating. Component survival on an unprotected satellite can occur if the component's melting temperature is sufficiently high and its shape is such that it loses heat fast enough to keep the temperature below the melting point of the structure. During reentry, the object starts decelerating and the loads on the structure can exceed up to 15g's. These loads combine with the high temperature hence the structure breaks apart. When the satellite components speed decrease, the rate of heating of structure reduces, therefore temperature decreases, and the objects starts to cool. Till then the objects have fallen to denser regions of the atmosphere and fall virtually straight down from the sky. They impact of space derbies to the ground is at relatively low speed, but it is hazardous to property and people.

It is difficult to locate where debris from a reentering satellite will hit on Earth because drag force on the object is proportional to density of the atmosphere and it varies in large magnitudes at high altitudes. In general, the estimated time that reentry will begin is within 5-8 percent of the actual time. Unfortunately, reentering objects travel with such a high speed that error in the time results to hundreds of miles on the ground. 
If a satellite or rocket body with propulsive capability, it can use its rocket motor to target the reentry into a desired area, such as the ocean. Objects in orbit are exposed to atmospheric drag, just as aircraft and automobiles are near the ground. In space the atmospheric drag is much less than that experienced closer to the ground but as satellite is continuously subjected to atmospheric drag even a small amount of drag can result in a satellite's reentry into the denser atmosphere.

Satellites orbiting at low altitudes could be removed from its orbit by atmospheric drag within a period of few months depending on the object and its altitude. Objects at higher altitudes may remain in orbit for hundreds or thousands of years. Some satellites and launch hardware have propulsive capability that can be used to deorbit these objects more quickly.

On reentering of satellite to atmosphere a large debris object will be subjected to extreme heating and loads caused because of the interaction of the high speed object with the atmosphere.At some locations on satellite, the temperature loads on the object reaches a critical point, aerodynamic loads reaches its maximum value resulting object to break. Breakup could be caused by the failure of critical structural components because of their high temperatures that exceed their melting points, in a more extreme case explosion of fuel or pressurized gas present in the object's tanks occurs. The object fragments into several smaller objects, and each of them continues to fragment or melt as long as sufficient heating and loads acts on them. When objects have slowed sufficiently, the heat rate decreases and debris falls and impact the ground.

Generally, about 10-30 percent of a satellite's mass will survive reentry. The actual percentage for a specific object depends on the strength of materials used for the object's construction and on shape, size, and weight of the reentering object. Consider an example, if the object is with empty fuel tanks made of stainless steel or titanium, both of which have high melting temperatures hence much of this material will survive. If much of the structure is made of aluminum material having low temperature of melting, a smaller percentage of it will survive thermal and aerodynamic load.

\section{Lagranginan Equation of motion under the influence of nonconservative forces}

Hamilton's principle lays the foundation of Lagrangian mechanics which is based on the concept of conservative forces in which the potential energy of interaction can be defined uniquely. In most of the cases non-conservative forces, such as friction and aerodynamic drag cannot be exempted from the analysis. It is important how to incorporate the effects of non-conservative forces in the Lagrange equations that describe the dynamical system in terms of generalized coordinates. Non-conservative forces are to be considered only when model is macroscopically large, the complex interactions between systems using empirical model. Empirical models of non-conservative forces are very useful in reducing the complexity of problems in the physical world. For this reason, it is required to find a way to incorporate them in the framework of Lagrangian mechanics. It is considered at first that evolution of a dynamical system in the absence of non conservative forces. Assuming that let there be $\mathrm{N}$ degrees of freedom in this problem can identify an appropriate set of $\mathrm{N}$ generalized coordinate's qj, $\mathrm{j}=1 \ldots \mathrm{N}$ that describe uniquely the dynamical state of the system. It is denoted by $\mathrm{xi}=$ $\mathrm{Xi}(\mathrm{qj}, \mathrm{t})$ the transformation rules between a set of Cartesian coordinates $\mathrm{xi}, \mathrm{i}=1, \ldots, \mathrm{N}$ and the generalized coordinates express the Lagrangian of the given system by $L(q j, q j)$ in terms of the latter coordinates and their time derivatives. The Euler-Lagrange equation for the system when non-conservative forces are absent is simply given by equation(1).

$$
\frac{\partial L}{\partial q_{i}}-\frac{d}{d t}\left(\frac{\partial L}{\partial \dot{q}_{i}}\right)=0 .
$$

Now considering the effect on this dynamical system of a non-conservative interaction.

Describes empirically by a Newtonian force with components $\mathrm{Fi}, \mathrm{i}=1, \ldots, \mathrm{N}$ in a Cartesian coordinate system.

Equations by a generalized force $\mathrm{Qj}, \mathrm{j}=1, \ldots, \mathrm{N}$ as

$$
\frac{\partial L}{\partial q_{i}}-\frac{d}{d t}\left(\frac{\partial L}{\partial \dot{q}_{i}}\right)=-Q_{j}
$$

Where the components of the generalized force are related to the Cartesian components of the nonconservative force, the transformation between Cartesian and generalized coordinates is by equation(3).

$$
Q_{j}=\sum_{i=1}^{3} F_{i} \frac{\partial X_{i}}{\partial q_{j}} \text {. }
$$

In the following application, using the methods of Lagrangian mechanics to investigate the influence of atmospheric drag on the motion of satellites in a low an Earth orbit is studied.

\section{The Reentry of Low Earth Orbit Satellite}

The most of artificial satellites are placed in orbits which are at relatively low distances from the Earth's surface. Theoretically, objects with distances smaller than about $1500 \mathrm{~km}$ from the Earth's surface are characterized under low Earth orbit satellites.

At distances as small as $100-300 \mathrm{~km}$, the density in the Earth's atmosphere is low as compared to that on the surface of Earth but it is enough to retard the motion of the satellites and this cause them to lose altitude and burn while approaching to the surface of Earth. The density and temperature in these layers of 
the atmosphere known as thermosphere depend strongly on the solar activity and changes adversely with solar activity. At the height of 100 miles (or 160 $\mathrm{km}$ ), the density of atmosphere is in the range of $10^{-9}$ $\mathrm{kg} m^{-3}$ and drops to $10^{-14}$ to $10^{-12} \mathrm{~kg} \mathrm{~m}-3$ at 300 miles (or $480 \mathrm{~km}$ ) which is very less value as on Earth surface. Goal in this section is to write the equations of motion for a satellite in low earth orbit considering into account the atmospheric drag. Given by equation(4).

$$
\vec{F}_{d}=-\frac{1}{2} \rho C_{a} A|\vec{u}| \vec{u},
$$

Where $\rho$ is the density of the atmosphere, Ca is the coefficient of aerodynamic drag. For the satellite, which depends on its exact shape, $A$ is the projected area of the satellite surface which is in the plane of perpendicular to its direction of motion, and $u$ is its velocity vector.

The satellites velocity vector, the drag force experienced on it and the Earth's gravitational acceleration $\mathrm{g}$ lie on a single plane that passes through the center of the Earth. This will be the plane of the satellite's orbit, since there is no interaction exists in the system that will force it to move away from that plane. Consider a set of Cartesian coordinates $\mathrm{x} 1$ and $\mathrm{x} 2$ on the orbital plane, with their origin at the center of the Earth, as well as a set of polar coordinates $r$ and $\phi$. The transformations between the Cartesian and polar coordinates are

$x_{1}=r \cos \phi$

$x_{2}=r \sin \phi$

And between the corresponding velocities are

$\dot{x}_{1}=\frac{\partial x_{1}}{\partial r} \dot{r}+\frac{\partial x_{1}}{\partial \phi} \dot{\phi}=-r \dot{\phi} \sin \phi+\dot{r} \cos \phi$

$\dot{x}_{2}=\frac{\partial x_{2}}{\partial r} \dot{r}+\frac{\partial x_{2}}{\partial \phi} \dot{\phi}=r \dot{\phi} \cos \phi+\dot{r} \sin \phi$

Using these transformations to write the kinetic energy of the satellite in terms of its generalized velocities as

$T=\frac{1}{2} m\left(\dot{x}_{1}^{2}+\dot{x}_{2}^{2}\right)=\frac{1}{2} m\left(r^{2} \dot{\phi}^{2}+\dot{r}^{2}\right)$,

Where $\mathrm{m}$ is the mass of the satellite. The use of the general expression for the potential energy in the Earth's gravitational field is given by equation (8). $V=-\frac{G M m}{r}$,

Where $M$ is the mass of the Earth, to write the Lagrangian of the system as shown in equation(9).

$L=T-V=\frac{1}{2} m\left(\dot{x}_{1}^{2}+\dot{x}_{2}^{2}\right)=\frac{1}{2} m\left(r^{2} \dot{\phi}^{2}+\dot{r}^{2}\right)+\frac{G M m}{r}$.

By applying the transformations between the Cartesian and generalized coordinates in order to derive the generalized components of aerodynamic drag forces starting from the Cartesian components of the Newtonian force

$$
\begin{aligned}
& F_{d, 1}=-\frac{1}{2} \rho C_{a} A\left(\dot{x}_{1}^{2}+\dot{x}_{2}^{2}\right)^{1 / 2} \dot{x}_{1} \\
& F_{d, 2}=-\frac{1}{2} \rho C_{a} A\left(\dot{x}_{1}^{2}+\dot{x}_{2}^{2}\right)^{1 / 2} \dot{x}_{2}
\end{aligned}
$$

After a small amount of algebraic simplification, it is found that

$$
\begin{aligned}
Q_{r} & =F_{1} \frac{\partial x_{1}}{\partial r}+F_{2} \frac{\partial x_{2}}{\partial r} \\
& =-\frac{1}{2} \rho C_{a} A\left(\dot{r}^{2}+r^{2} \dot{\phi}^{2}\right)^{1 / 2} \dot{r}
\end{aligned}
$$

And

$$
\begin{aligned}
Q_{\phi} & =F_{1} \frac{\partial x_{1}}{\partial \phi}+F_{2} \frac{\partial x_{2}}{\partial \phi}(14) \\
& =-\frac{1}{2} \rho C_{a} A\left(\dot{r}^{2}+r^{2} \dot{\phi}^{2}\right)^{1 / 2} r^{2} \dot{\phi}
\end{aligned}
$$

Inserting this expression into the Lagrange equations of motions, it gets simplified as

$$
\begin{aligned}
& \frac{\partial L}{\partial r}-\frac{d}{d t}\left(\frac{\partial L}{\partial \dot{r}}\right)=-Q_{r} \\
& \Rightarrow m r \dot{\phi}^{2}-m \ddot{r}-\frac{G M m}{r^{2}}=\frac{1}{2} \rho C_{a} A\left(\dot{r}^{2}+r^{2} \dot{\phi}^{2}\right) \dot{r}
\end{aligned}
$$

And

$\frac{\partial L}{\partial \phi}-\frac{d}{d t}\left(\frac{\partial L}{\partial \dot{\phi}}\right)=-Q_{\phi}$

$\Rightarrow-m r^{2} \ddot{\phi}-2 m r \dot{r} \dot{\phi}=\frac{1}{2} \rho C_{a} A\left(\dot{r}^{2}+r^{2} \dot{\phi}^{2}\right)^{1 / 2} r^{2} \dot{\phi}$.

Rearranging terms and dividing both sides of the above two equation by the mass ' $m$ ' of the satellite results to

$\ddot{r}-r \dot{\phi}^{2}=-\frac{G M}{r^{2}}-\frac{1}{2}\left(\frac{C_{a} A}{m}\right) \rho\left(\dot{r}^{2}+r^{2} \dot{\phi}^{2}\right)^{1 / 2}$

$r \ddot{\phi}+2 \dot{r} \dot{\phi}=\frac{1}{2}\left(\frac{C_{a} A}{m}\right) \rho\left(\dot{r}^{2}+r^{2} \dot{\phi}^{2}\right)^{1 / 2} r \dot{\phi}$.

These above two equations are coupled second-order differential equations in time for the two generalized coordinates $r$ and $\phi$. These equations can be integrated easily using computer, knowing the two components of the position and the velocity of the satellite as initial conditions.

The satellite mostly revolves on the circular orbit, while slowly drifting and coming closer towards the Earth's surface. Consider P is the orbital period of the satellite at any given altitude and by equation (22).

$T \equiv\left|\frac{r}{\dot{r}}\right| \quad$,

The characteristic timescale for reentry, the above assumptions imply that $\tau>>P$. In the radial equation of motion, i.e., equation (20), the drag force is 
negligible compared to the gravitational force, by assumption. And, therefore, the radial acceleration " $r$ term is considered to be negligible. Hence equation(20) becomes simply to

$$
\dot{\phi}^{2} \square \frac{G M}{r^{3}} \text {, }
$$

This is the usual expression for the angular velocity of a satellite in orbit. On calculating the angular deceleration of satellite by taking first derivative of angular velocity ,i.e., equation(23) we get,

$\ddot{\phi}=\frac{d \dot{\phi}}{d t} \square \frac{d}{d t}\left(\frac{G M}{r^{3}}\right)^{1 / 2}=-\frac{3}{2}\left(\frac{G M}{r^{3}}\right)^{1 / 2} \frac{\dot{r}}{r}$

Satellite retardation because of reentry take long time as this is very slow steady process. Hence it is convenient to assume that the angular retardation and radial retardation is almost negligible when compared to change in angular velocity and radial distance because of atmospheric drag.

Therefore equation (20) and equation (21) reduces to $r \dot{\phi}^{2}=\frac{G M}{r^{2}}$

$\dot{r} \dot{\phi}=\frac{1}{4}\left(\frac{C_{a} A}{m}\right) \rho\left(\dot{r}^{2}+r^{2} \dot{\phi}^{2}\right)^{1 / 2} r \dot{\phi}$.

On squaring the above equation and after simplification we get

$\dot{r}^{2} \dot{\phi}^{2}=\frac{1}{16}\left(\frac{C_{a} A}{m}\right)^{2} \rho^{2}\left(\dot{r}^{2}+r^{2} \dot{\phi}^{2}\right) r^{2} \dot{\phi}^{2}$.

On further simplification we get

$\frac{\dot{r}^{2}}{r^{2}}=\frac{1}{16}\left(\frac{C_{a} A}{m}\right)^{2} \rho^{2}\left(\dot{r}^{2}+r^{2} \dot{\phi}^{2}\right)$.

Let us assume

$f=\frac{1}{16}\left(\frac{C_{a} A}{m}\right)^{2} \rho^{2}$.

Therefore equation(28) becomes

$\frac{\dot{r}^{2}\left(1-f r^{2}\right)}{r^{2}}=r^{2} \dot{\phi}^{2} f$

From equation(22) on simplying above equation we get

$\frac{1}{T^{2}}=\frac{r^{2} \dot{\phi}^{2} f}{1-f r^{2}}$
The final characteristic equation for reentry rate calculation is

$T=\frac{1}{C_{a} A \rho} \sqrt{\frac{r\left(16 m^{2}-C_{a}^{2} A^{2} \rho^{2} r^{2}\right)}{G M}}$

The above timescale characteristic equation depicts the reentry time calculation of the satellite orbiting around the Earth considering coefficient of drag (Ca), density at the satellite orbit $(\rho)$, projected area of the satellite (A) and the radial distance between satellite orbit and centre of the Earth(r).The above equation enables to predict the time after which the satellite reenters the Earth's atmosphere.

\section{Conclusions}

The reentry characteristic timescale equation relates as the distance from centre of the earth increases, reentry time increases. Therefore satellites at higher altitude are less prone to reentry phenomenon; hence reentry is concerned with low earth orbiting satellite. The determination of the rate of reentry of any space junk, space debris or satellite can be calculated using Lagrangian Mechanics. Objects orbiting at low altitudes may be removed from orbit by atmospheric drag within few weeks to months or years depending on the object and its altitude. The risk of the reentry of satellite can be estimated and the time when the satellites with inbuilt propellant or small engine are to be backing fired into their original orbit can be calculated

\section{References}

King-Hele, D.G., 1962 The Contraction of Satellite Orbits Under the Influence of Air -- Drag, IIl. High-eccentricity orbits, Proceedings of The Royal Society, Series A, Vol.267, . No.1331,

De Lafontaine J, and Garg S.C., Jun. 1980A Review of Satellite Lifetime and Orbit Decay Prediction, Institute for Aerospace Studies, University of Toronto, UTIAS, Review No.43,.

King-Hele, D.G., 1977 Methods for Predicting Satellite Orbital Lifetimes, Royal Aircraft • Establishment, TR 77111,.

Reflin 0, Stem, R. and Pitz C,Review of Orbital Reentry Risk Predictions, 1992.ATR-92(2835)-1, The Aerospace Cot poration, El Segundo, $C A$,

Preliminary Design of Small Satellites for Atmospheric Reentry Derek J. Dalle and Sara C. Spangelo University of Michigan Ann Arbor, Michigan 48105

Gallais P(2007) : Atmospheric re-entry vehicle mechanics. Springer

Galley, Chad R. (2013). Classical Mechanics of Nonconservative Systems. Physical Review Letters 110 (17): 174301. 